\title{
SEGMENTATION ON SENTINEL-3 DATA FOR SURFACE HEAT ISLAND DETECTION
}

\author{
I. Kotaridis ${ }^{1} *$, M. Lazaridou ${ }^{1}$ \\ ${ }^{1}$ Aristotle University of Thessaloniki, Faculty of Engineering, School of Civil Engineering, \\ Lab. of Photogrammetry - Remote Sensing, 54124 Thessaloniki, Greece - (iskotarid, lazamari)@civil.auth.gr
}

Commission III, WG III/1

KEY WORDS: Land surface temperature, heat island, image segmentation, thermal infrared remote sensing, NDVI, Sentinel-3.

\begin{abstract}
:
The commonly higher temperatures in urban environment, compared to its surrounding countryside, have been observed and described for a long time. Several studies, focusing on the quantification of this phenomenon, have been carried out. Detecting, understanding and monitoring of heat islands is of utmost importance. This paper presents a methodological framework for a rapid identification of surface heat islands. For this purpose, image pre-processing, image segmentation and image analysis are conducted in SNAP, Orfeo ToolBox (OTB) and QGIS accordingly. Sentinel-3 data were obtained and land surface temperature (LST) product was utilized. This is not equal to air temperature that is presented in the daily weather report; however, it is a quite good and accessible indicator. Specifically, two products were used, one of day observation and one of night observation in order to highlight the differentiation of these two views. In addition, the correlation between NDVI and LST was examined in order to comprehend how land cover affects temperature. The proposed methodology was carried out by obtaining freely-available data that were processed in open-source software.
\end{abstract}

\section{INTRODUCTION}

\subsection{Surface heat islands}

It is a fact that many people live in urban areas, with their number estimated to rapidly escalate in the near future (Sobrino and Irakulis, 2020). The change of vegetation land cover to built-up areas significantly affects the energy balance. Impervious surfaces and heat from human activities cause the increase of urban heat island that is the difference between the air temperature within the urban environment and the air temperature of its surroundings (Amorim, 2018). In general, the temperature is higher in urban areas than in rural areas, particularly at night. This circumstance plays an important role in people's psychology and economic activities. Thus, monitoring heat islands is of great importance (Sobrino and Irakulis, 2020). When monitoring is carried out through remote sensing TIR data, the parameter that is analyzed is the land surface temperature (Weng, 2009; Sobrino and Irakulis, 2020).

Land surface temperature is a significant feature in the analysis of urban thermal environment and dynamics. It adjusts the air temperature of the lower layer of urban environment and is the most important factor in calculating surface radiation and energy exchange, the indoor climate of buildings and human thermal comfort conditions in urban areas. Decisive factors that affect land surface temperatures include the physical attributes of different types of urban surfaces, the geometry of streets, the sky view factor, the traffic loads and anthropogenic activities (Weng, 2009).

The commonly higher temperatures in urban environment, compared to its surrounding countryside, have been observed and described for a long time (Chen et al., 2006). Several studies have been carried out that focus on the quantification of this phenomenon. However, it is also important to investigate land surface temperatures in a broader scale. Detecting, understanding and monitoring surface heat islands is of utmost importance. Remotely sensed thermal infrared (TIR) data comprise an important source of information for this purpose. Considering that close relationships between near surface air temperatures and land surface temperatures have been calculated, surface heat island is a reliable indicator for further analysis.

\subsection{Image segmentation}

Image segmentation is the initial and integral part in order to produce the fundamental elements of Object-Based Image Analysis (OBIA, Cheng and Han, 2016). It includes the partitioning of an imagery into spatially adjoining and homogenous regions (segments) that constitute the foundation for further analysis (Blaschke, 2004; Nussbaum and Menz 2008). The aim of image analysis study affects the selection of segmentation algorithm. However, it is generally accepted that there is no perfect image segmentation algorithm that will suit every satellite image. In addition, it has to be mentioned that most segmentation algorithms do not directly obtain meaningful image objects, but clusters with generic labels (Thenkabail, 2015). Finally, a fairly challenging task in image segmentation procedure is to determine the optimal segmentation parameters' values in order to produce segments that will comply with user requirements and objective of the study (Cheng and Han, 2016). Even today, segmentation continues to be a significantly interactive procedure that is characterized by trial-and-error approach.

Image segmentation methods can be separated into spectrallybased approaches that include thresholding-based and feature space clustering-based algorithms and spatially-based

\footnotetext{
* Corresponding author
} 
approaches that include edge-based, region-based and graphbased algorithms. In addition, a few hybrid methodologies have been developed in order to exploit the advantages of the aforementioned algorithms. Despite their conceptual differences, all these methods share a common characteristic, the focus on extracting homogenous regions (Kotaridis and Lazaridou, 2021).

\subsection{Relevant studies}

Several researchers have obtained satellite data and implemented a remote sensing methodology to extract heat islands. In particular, $\mathrm{Li}$ et al. (2009) assessed the impact of urban growth by utilizing GIS and remote sensing techniques in order to quantify the change in land surface temperature of Shanghai. For this reason, they used the Landsat TM thermal TIR band. In the same manner, Klok et al. (2012) employed thermal infrared Landsat satellite data to spatially quantify the surface heat island of Rotterdam. In order to achieve that, they compared the surface temperature within the city to the rural surface temperature of the surroundings. Sobrino et al. (2013) evaluated the surface urban heat island in the city of Madrid. Peres et al. (2018) studied the urban heat island in the Metropolitan Area of Rio de Janeiro. They analyzed the land surface temperature and land use patterns with the use of Landsat-5, Landsat-7 and Landsat- 8 data within a 32-year time span. Shirani-bidabadi et al., (2019) carried out a study in order to analyze the thermal levels within a city in Iran. For this reason, they retrieved the land surface temperature form Landsat 7 and Landsat 8 satellite data. Simanjuntak et al. (2019) implemented and object-based image analysis in order to map local climate zones in a city of Indonesia. These zones can be really valuable when investigating land use dependent thermal patterns. So, they analyzed their relationship with land surface temperature. Finally, Wang et al. (2019) obtained Landsat 8 data in order to analyze the relationship between land cover type and land surface temperature within the city of Shenzhen, China.

\subsection{Scope of the study}

The primary objective of this study is to present an efficient methodological framework for the rapid identification of surface heat islands in an on administrative entity including not only the urban environment but also the suburban environment and the provinces. For this reason, only freely available satellite data and software were employed. The methodology presented can be effectively used in future surface heat island detection studies.

\section{STUDY AREA AND SATELLITE DATA}

\subsection{Study area}

The region of interest that was selected for this study covers an area of $3680 \mathrm{~km}^{2}$. It is located in the north part of mainland Greece (Figure 1). It comprises of several diverse land cover types including concrete, metal, asphalt, water, vegetation and agricultural areas. In specific, the borders of Thessaloniki regional unit were selected as the boundaries of the study area. It includes Lake Koroneia that is located about $14 \mathrm{~km}$ east of Thessaloniki city center and Lake Volvi, which is the second largest lake in the country. In addition, some mountainous areas can be detected in the central and north part of the regional unit.

\subsection{Satellite data}

In 2016, Sentinel-3 mission was developed in order to support Copernicus ocean, land, atmospheric, emergency, security and cryospheric applications. It is jointly operated by the European Space Agency (ESA) and European Organisation for the Exploitation of Meteorological Satellites (EUMETSAT). Among the main objectives of the mission is the measurement

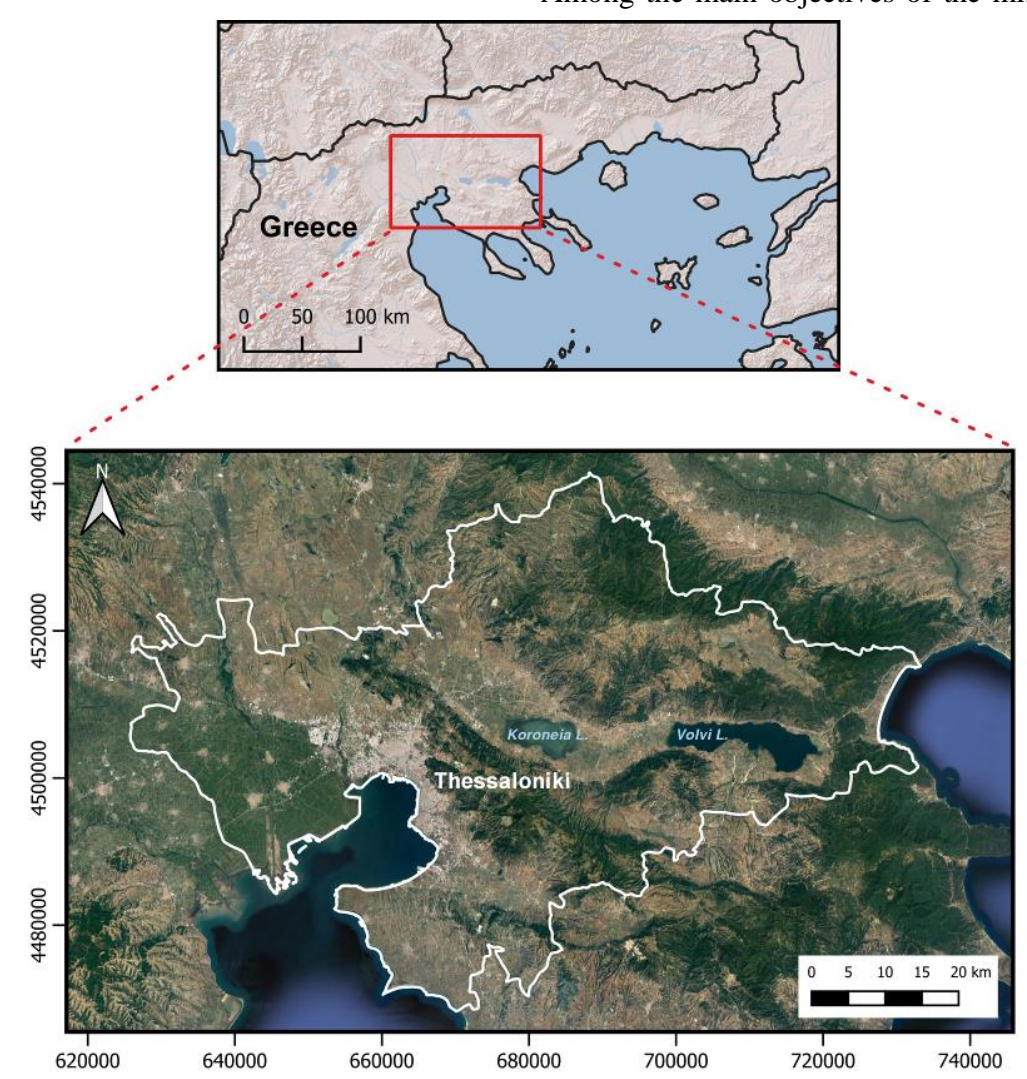

Figure 1. Study area located in Greece (upper part) and the subset that is illustrated in Google basemap (lower part) (Source: Google Maps, modified by the author). 
of land surface temperature through the Sea and Land Surface Temperature Instrument (SLSTR, ESA, 2020a).

In this study, two Sentinel-3B clear sky products were obtained that include thermal infrared bands. The scenes acquisition date is 12 August 2019. The land surface temperature (LST) product was utilized for the analysis. This is not equal to air temperature that is presented in the daily weather report; however, it is a quite good and accessible indicator. In specific, two scenes were used, one of day observation and one of night observation in order to highlight the differentiation of these two views. A subset was extracted from the main scene for analysis in order to include the Regional Unit of Thessaloniki.

\begin{tabular}{ccc}
\hline Product & Acquisition time & Resolution (m) \\
\hline S3B_SL_2_LST & 2019-08-19T09:00:20 & 1000 \\
S3B_SL_2_LST & 2019-08-19T19:06:15 & 1000 \\
\hline
\end{tabular}

Table 1. Sentinel-3 data used in this study.

\section{METHODOLOGICAL PROCEDURE}

The methodology that was implemented in order to identify and extract surface heat islands in the study area is presented in Figure 2.

\subsection{Tools}

Sentinel Application Platform (SNAP) and QGIS were used for digital processing of the sentinel-3 data. SNAP is an opensource architecture that is optimal for earth observation processing and analysis (ESA, 2020b). QGIS is a free and opensource Geographic Information System that supports the creation, editing, visualization, and publication of geospatial data (QGIS, 2020).

\subsection{Initial processing of data}

First, initial processing of Sentinel-3 data was carried out in SNAP. It includes a preliminary subset to focus on an area around the regional unit of Thessaloniki that will be used for the study. This step reduces the size of the image and as a consequence the processing time. In order to apply this spatial subset, geographic coordinates were specified. In addition, reprojection was conducted to the subset Sentinel-3 product. This process produces an output in a projected coordinate system (WGS 84/UTM zone 34N). Following the reprojection of the data, some distortions were observed at the edges. In order to solve this issue, another spatial subset was carried out. Finally, the LST band was exported as a raster file in GeoTIFF format (Figure 3).

The LST raster files that was created according to the aforementioned steps were added in QGIS. For the purpose of this study, each file was clipped to the boundaries of the regional unit of Thessaloniki. The resolution of the input raster was not altered and a mask layer was provided to complete the clipping of the image.

\subsection{Interpretation of derived temperature maps}

Following the implementation of initial processing to both Sentinel-3 products (day and night scene), visual interpretation was carried out of the LST images that are presented in Figure 4 and Figure 5.
As observed in LST map of day observation (Figure 4), the derived output shows a high temperature dispersion. High temperature values are noticed throughout the study area, except for water bodies and green areas. On the other hand, as observed in LST map of night observation (Figure 5), some clusters seem to formulate. Now, warmer and colder parts of the study area can be clearly identified. Low LST values are observed in mountainous areas (where high elevation leads to lower temperatures). In addition, the impact of lakes and "green" areas can be recognized. On the other hand, looking at the warm spots, the location of the city of Thessaloniki can be clearly identified, since dry impervious surfaces absorb sunlight more easily and become hotter in comparison with moist surfaces.

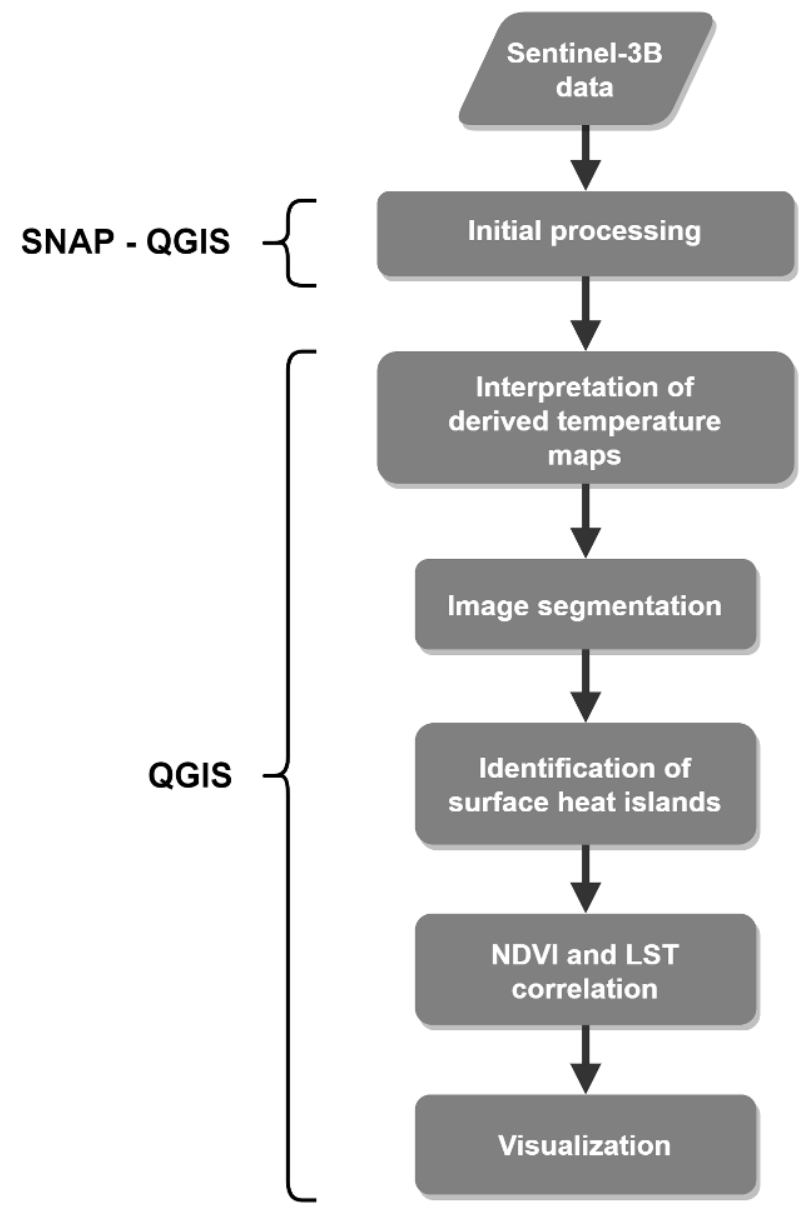

Figure 2. Schematic diagram of the proposed methodological framework.

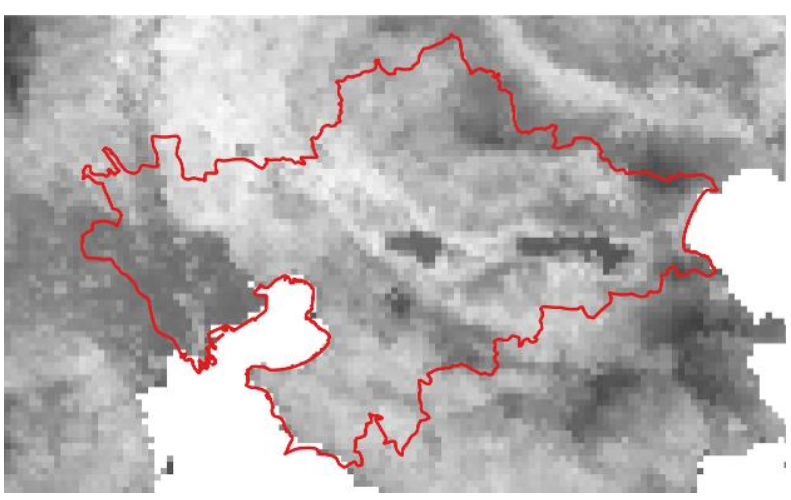

Figure 3. Land surface temperature band of day observation, after the initial processing in SNAP. 


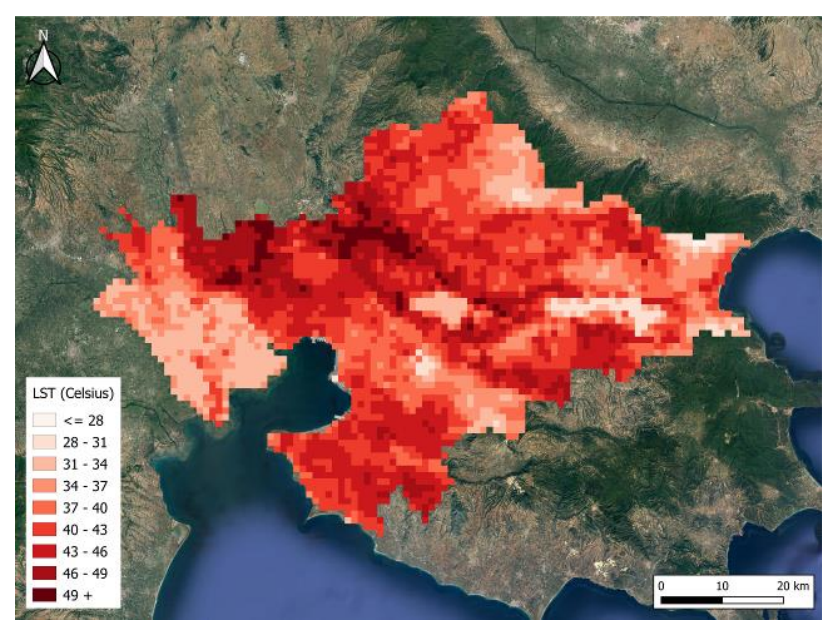

Figure 4. Land surface temperature map of day observation.

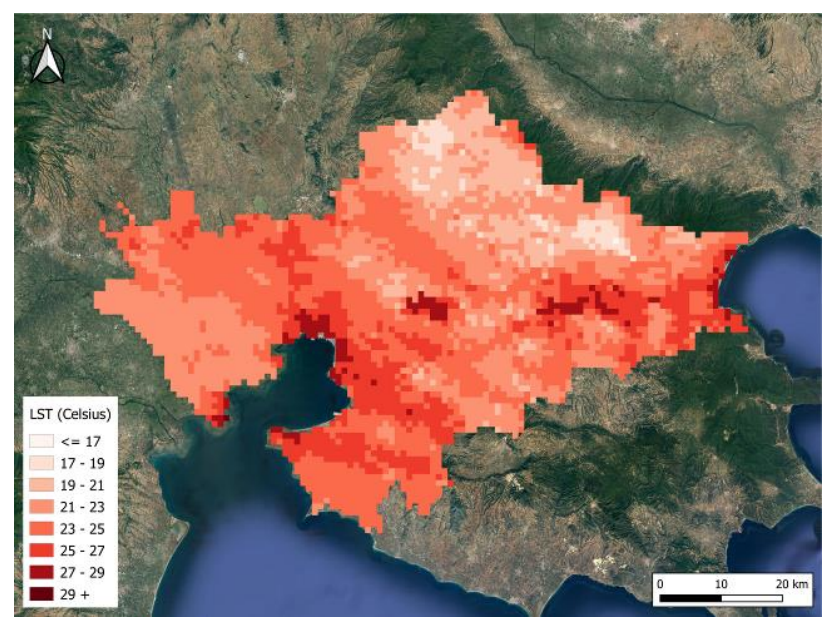

Figure 5. Land surface temperature map of night observation.

\subsection{Image segmentation}

The produced LST raster files from the initial processing of the Sentinel-3 data were used as input for the segmentation procedure. Image segmentation was carried out in Terminus QGIS plugin (Kotaridis and Lazaridou, 2020). It is a fast and easy to use plugin that allows users to implement image segmentation on remote sensing data.

Terminus includes a few popular image segmentation algorithms: felzenszwalb, quick shift, slic and watershed. Each algorithm produces two outputs, a vector file and a raster file. The plugin offers user the option to compute various statistics over each segment. If this is the case, these zonal statistics are included in the fields of the output vector file and a multiband raster file is created.

In this study, following several trial-and-error attempts, felzenszwalb image segmentation algorithm was employed and the mean value zonal statistic was included in the fields of the output vector file. The extracted segments for both day and night observation are presented in Figure 6 and Figure 7. The symbology applied is based on the mean LST value of each segment.

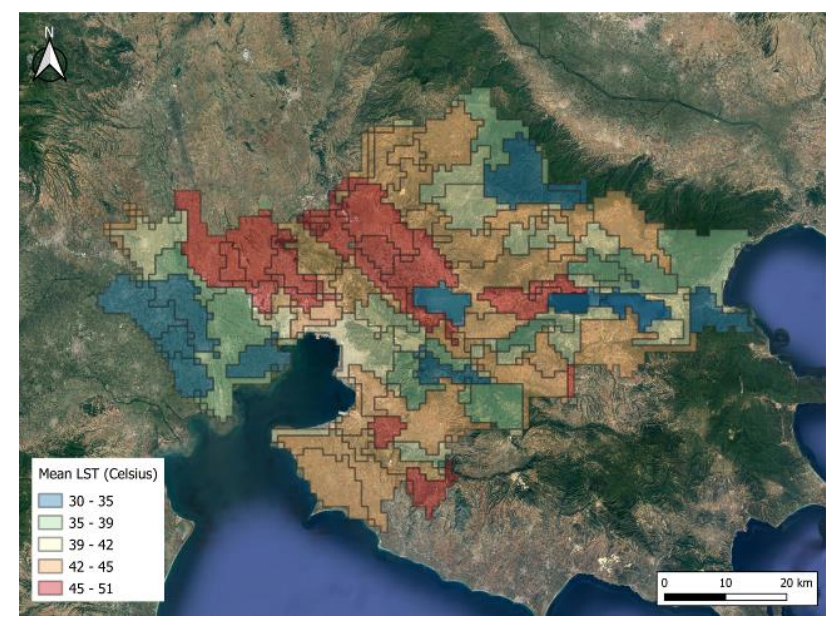

Figure 6. Mean LST segments of day observation.

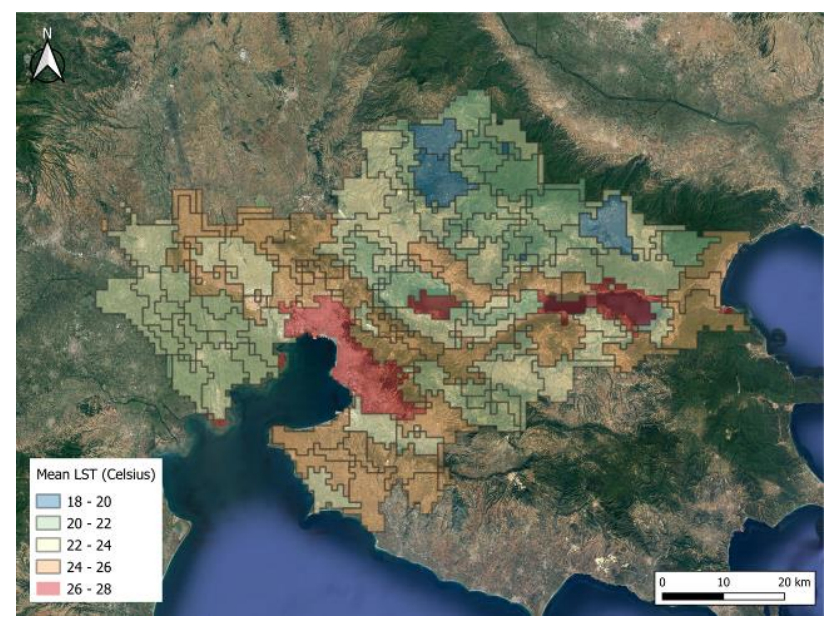

Figure 7. Mean LST segments of night observation.

\subsection{Identification of surface heat islands}

Since the definition of heat island is the presence of an area warmer than its surrounding landscape, they can be developed on both urban and rural areas. However, it is a fact that the most studied heat islands are those that refer to the urban environment, due to the fact that they constitute a risk for the human wellness. Regarding the presence of heat islands in the study area, a clearer view of the situation can be obtained by observing the land surface temperature segments of the night observation. A few segments with higher temperature can be identified in the center and the east part of the study area. They mostly include part of the built-up environment and lakes, as expected. The heat island phenomenon has been generally related to urban environment, due to the fact that its surfaces are characterized by low albedo high and advantageous thermal properties for energy storage and heat release. These elements are responsible for the increased temperatures of built-up areas in comparison with their rural more vegetated provinces. The fact that water segments are appear in dark red is related to the fact that the land cools off quickly, but the surface of water remains warm since water loses and gains heat at a slower rate. In addition, agricultural areas are characterized by higher LST values in comparison with "green" and hilly regions. Considering that the temperature in urban areas is higher than in rural areas, particularly at night, the extraction of heat islands was carried out on the image of night observation. 
The segments with the highest land surface temperature values in comparison with their surrounding landscape indicate the presence of surface heat islands, thus they were extracted and are presented in Figure 8. They include both the urban environment as well as two water bodies. Whilst the former is caused by human intervention, the latter is a natural phenomenon. It has to be mentioned that the segment of urban environment matches very well with the outline of the inhabited area of the city.

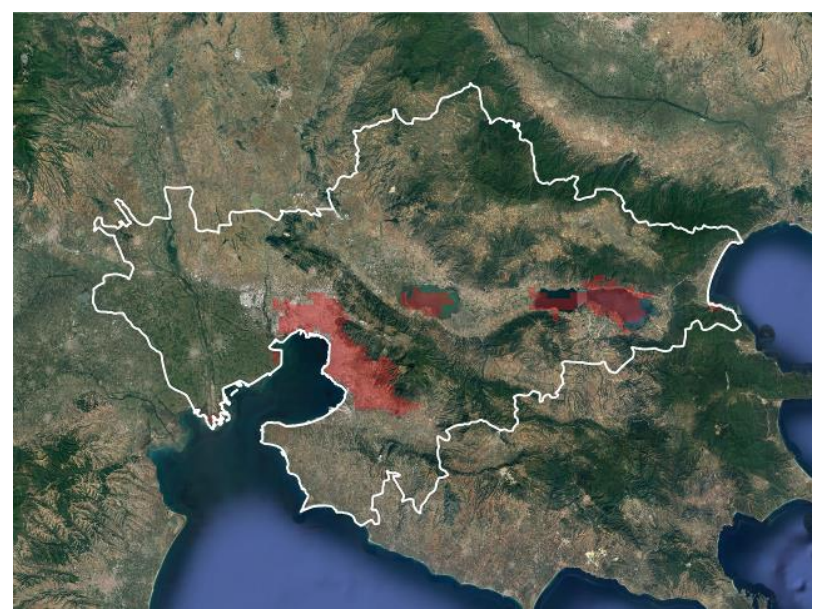

Figure 8. The segments with the highest land surface temperature values.

\subsection{NDVI and LST correlation}

Precise and timely extraction of land cover types is crucial for environmental management and monitoring (Wang et al., 2019). In environmental studies, it is a common procedure to analyze land surface temperature patterns and their relationship with surface characteristics (Weng, 2009). In particular, the transition from rural landscape to built-up impervious surfaces can substantially affect LST (Wang et al., 2019).

The understanding of how different land cover types affect the distribution of LST are very useful in order to interpret the mechanisms that are responsible for the development of heat islands. Remote sensing techniques can be utilized to investigate variations in heat island phenomena among separate land cover types. Thus, they constitute a valuable tool in monitoring the spatiotemporal changes of heat islands (Wang et al., 2019). In his study, the correlation between NDVI (Figure 9) and LST was examined across a line of interest that intersects several diverse land cover types (Figure 10). The results are presented in a form of a transect plot of these two features for both day and night observation (Figures 11 and 12).

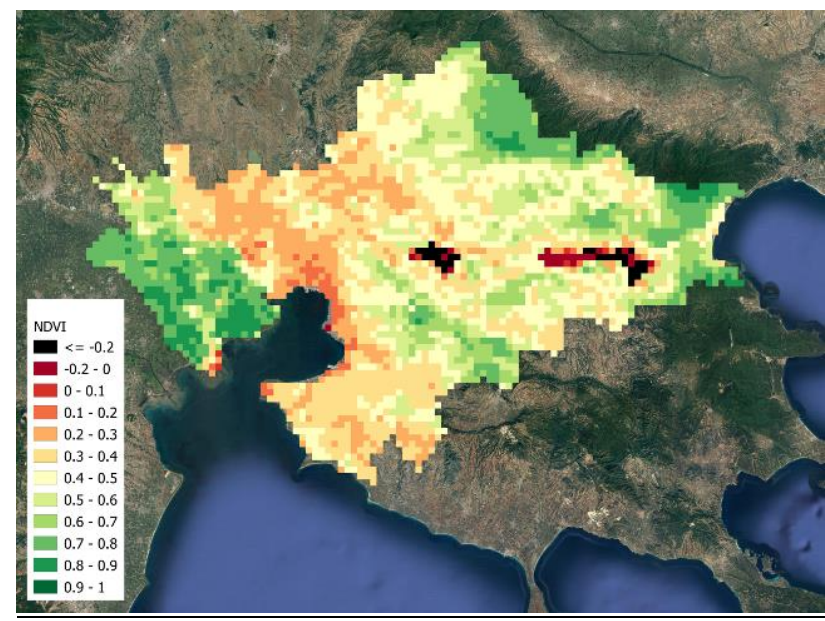

Figure 9. NDVI of the study area.

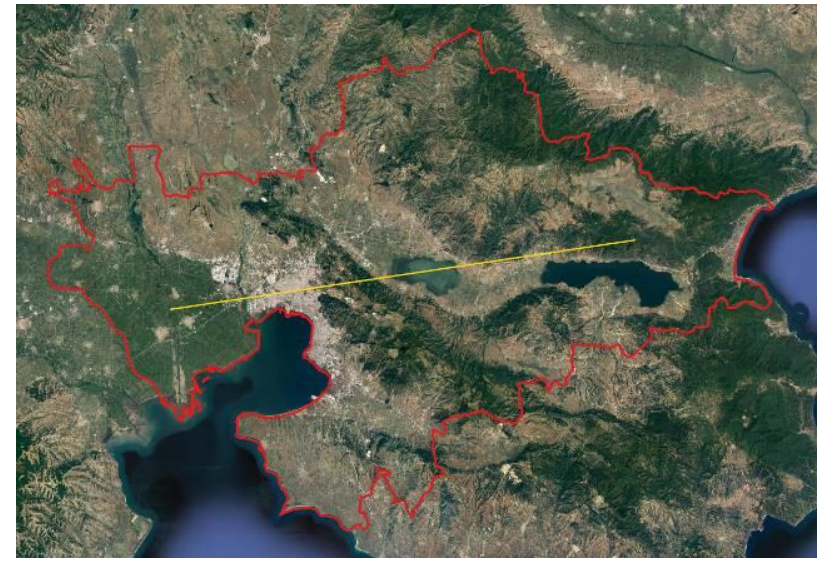

Figure 10. Line of interest.

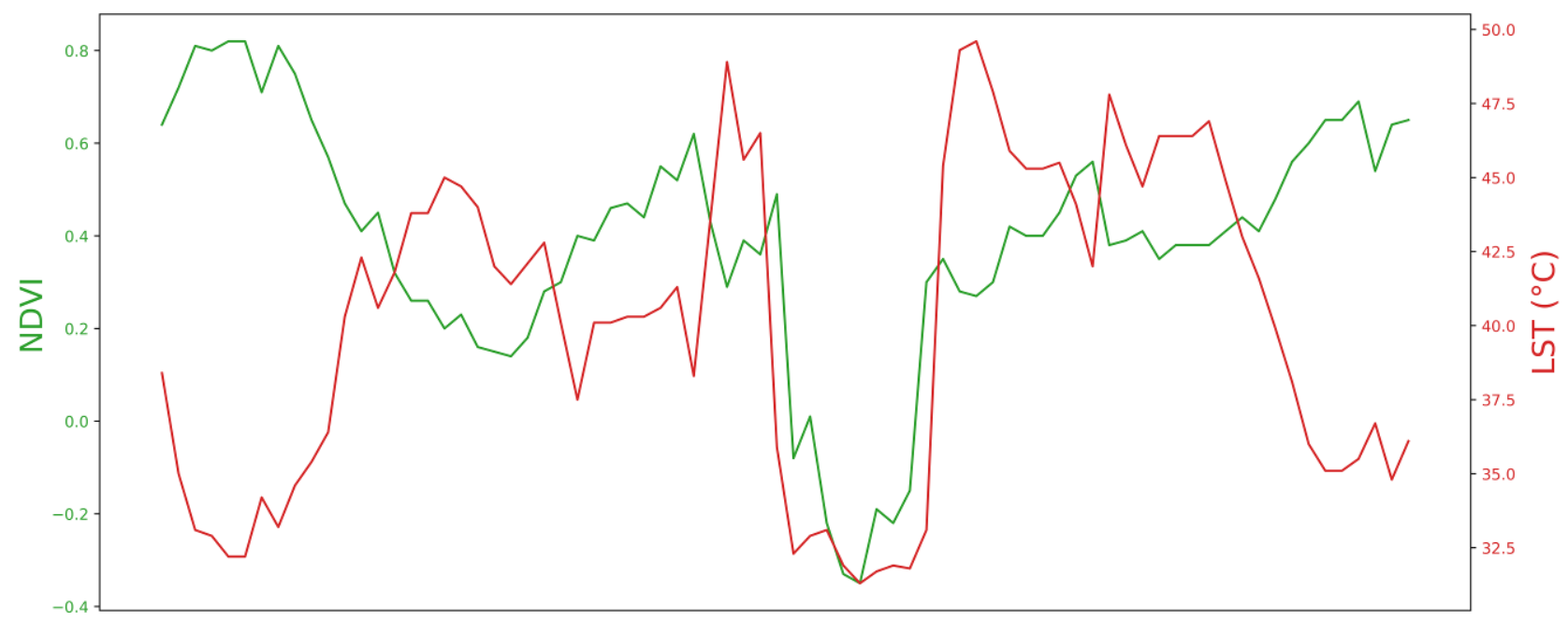

Figure 11. The correlation between NDVI and LST of day observation. 


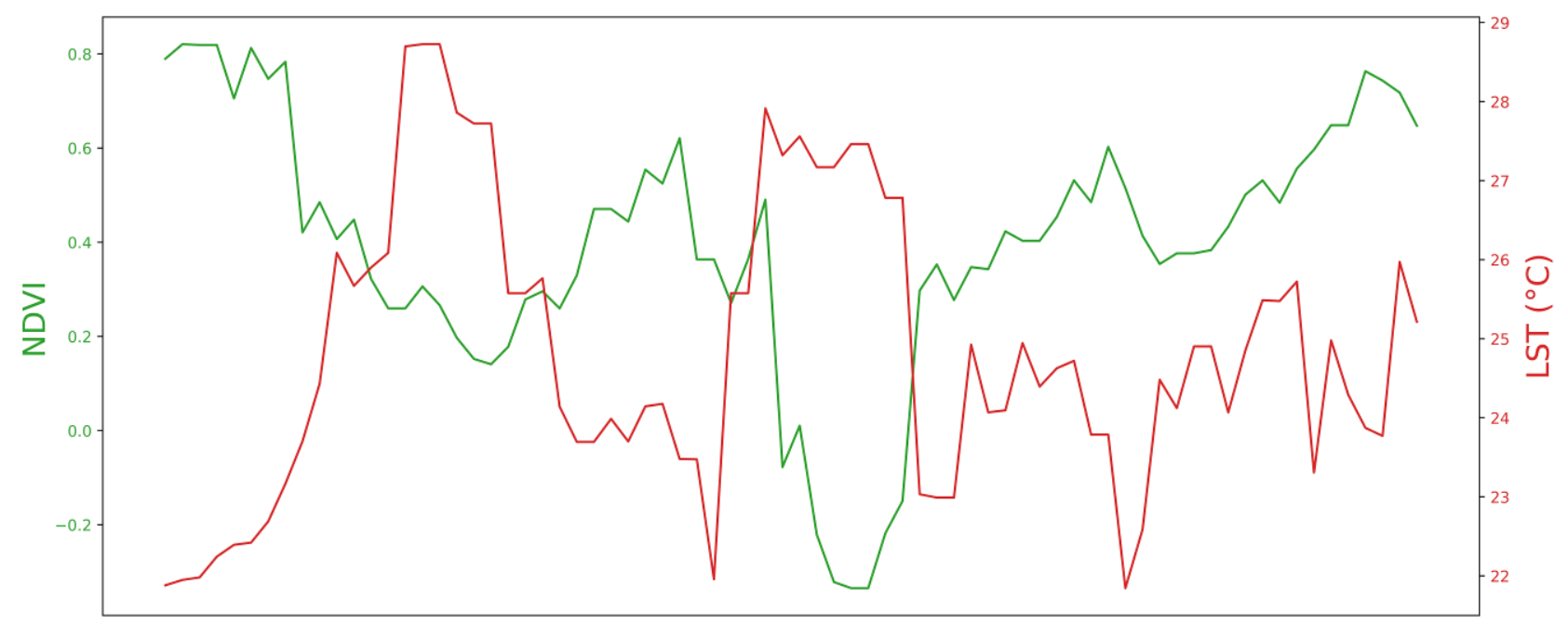

Figure 12. The correlation between NDVI and LST of night observation.

\section{DISCUSSION}

It has to be underlined that in situ air temperature measurements struggle to monitor the spatial extent of surface heat islands. Single in situ measurements refer to local conditions. Considering that high density measurement networks are expensive, thermal infrared remote sensing data and methods have been employed in several studies to evaluate the spatiotemporal distribution of surface heat islands.

The methodological framework presented in this study includes the implementation of an object-based approach and GIS techniques to detect and obtain local surface heat islands. The integration of Sentinel-3 data in this framework facilitates an efficient and fast extraction of heat islands. This information is valuable in order to identify warning situations and develop measures. The implemented approach depicted that it is feasible to obtain precise results in a timely manner by obtaining freelyavailable data that are processed in open-source software.

As shown in the produced correlation graphs, LST values related to vegetated areas are relatively low in both day and night observation. Water surfaces correspond to low LST values in the day and high LST values in the night. Regarding the built-up areas that include residential, commercial and industrial regions, manly consisting of impervious materials, higher LST values are observed during the night. This feature facilitates the detection of surface heat islands.

The methodology presented and applied works quite well for the detection of surface heat islands in a specific scene. However, when a more in-depth analysis is needed, several LST products of the study area should be processed and evaluated in order to investigate the development of heat islands in a temporal manner. Since Sentinel-3 mission delivers products from 2016, it will be a valuable source of data for future studies.

Finally, it has to be mentioned that the proposed methodology is appropriate for detecting local surface heat islands and observing the effect of different land cover on LST at larger geographical scales. However, urban planners also investigate heat islands within the urban environment. Such a detailed analysis would require data with a higher spatial resolution, such as Landat- 8 data.

\section{CONCLUSION}

In this study, a methodology for rapid detection of surface heat islands and quite accurate delineation of their boundaries is presented, using both freely-available data and open-source software. For this reason, Sentinel-3 data were obtained covering the area of interest. Following the initial processing of the data, LST temperature maps of day and night observation were produced. Next, image segmentation was carried out in order to extract homogeneous clusters. The segments with the highest mean LST values were isolated to extract the surface heat islands of the study area. Finally, the relationship between LST and NDVI was presented.

\section{REFERENCES}

Amorim, M.C. de C.T., 2018. Spatial variability and intensity frequency of surface heat island in a Brazilian city with continental tropical climate through remote sensing. Remote Sensing Applications: Society and Environment 9, 10-16. doi.org/10.1016/j.rsase.2017.11.001

Blaschke, T., Burnett, C., Pekkarinen, A., 2004. Image Segmentation Methods for Object-based Analysis and Classification, in: Jong, S.M.D., Meer, F.D.V. (Eds.), Remote Sensing Image Analysis: Including the Spatial Domain. Springer, Dordrecht, pp. 211-236. doi.org/10.1007/1-40202560-2_12

Chen, X.-L., Zhao, H.-M., Li, P.-X., Yin, Z.-Y., 2006. Remote sensing image-based analysis of the relationship between urban heat island and land use/cover changes. Remote Sensing of Environment 104, 133-146. doi.org/10.1016/j.rse.2005.11.016

Cheng, G., Han, J., 2016. A survey on object detection in optical remote sensing images. ISPRS Journal of Photogrammetry and Remote Sensing 117, 11-28. doi.org/10.1016/j.isprsjprs.2016.03.014

ESA, 2020a. SENTINEL-3.

https://sentinel.esa.int/web/sentinel/missions/sentinel-

3/overview (30 March 2021)

ESA, 2020b. SNAP. https://step.esa.int/main/toolboxes/snap/ (30 March 2021). 
Klok, L., Zwart, S., Verhagen, H., Mauri, E., 2012. The surface heat island of Rotterdam and its relationship with urban surface characteristics. Resources, Conservation and Recycling 64, 23 29. doi.org/10.1016/j.resconrec.2012.01.009

Kotaridis, I., Lazaridou, M., 2021. Remote sensing image segmentation advances: A meta-analysis. ISPRS Journal of Photogrammetry and Remote Sensing 173, 309-322. https://doi.org/10.1016/j.isprsjprs.2021.01.020

Kotaridis, I., Lazaridou, M., 2020. Terminus. https://github.com/ikotarid/Terminus

Li, J., Wang, Xiang-rong, Wang, Xin-jun, Ma, W., Zhang, H., 2009. Remote sensing evaluation of urban heat island and its spatial pattern of the Shanghai metropolitan area, China. Ecological Complexity 6, 413-420. doi.org/10.1016/j.ecocom.2009.02.002

Nussbaum, S., Menz, G., 2008. Object-based image analysis and treaty verification: new approaches in remote sensing applied to nuclear facilities in Iran. Springer, New York, NY.

Peres, L. de F., Lucena, A.J. de, Rotunno Filho, O.C., França, J.R. de A., 2018. The urban heat island in Rio de Janeiro, Brazil, in the last 30 years using remote sensing data. International Journal of Applied Earth Observation and Geoinformation 64, 104-116. doi.org/10.1016/j.jag.2017.08.012

QGIS, 2020. A Free and Open Source Geographic Information System. https://qgis.org/en/site/ (10 September 2020).

Shirani-bidabadi, N., Nasrabadi, T., Faryadi, S., Larijani, A., Shadman Roodposhti, M., 2019. Evaluating the spatial distribution and the intensity of urban heat island using remote sensing, case study of Isfahan city in Iran. Sustainable Cities and Society 45, 686-692. doi.org/10.1016/j.scs.2018.12.005

Simanjuntak, R.M., Kuffer, M., Reckien, D., 2019. Objectbased image analysis to map local climate zones: The case of Bandung, Indonesia. Applied Geography 106, 108-121. doi.org/10.1016/j.apgeog.2019.04.001

Sobrino, J.A., Irakulis, I., 2020. A Methodology for Comparing the Surface Urban Heat Island in Selected Urban Agglomerations Around the World from Sentinel-3 SLSTR Data. Remote Sensing 12, 2052. doi.org/10.3390/rs12122052

Sobrino, J.A., Oltra-Carrió, R., Sòria, G., Jiménez-Muñoz, J.C., Franch, B., Hidalgo, V., Mattar, C., Julien, Y., Cuenca, J., Romaguera, M., Gómez, J.A., De Miguel, E., Bianchi, R., Paganini, M., 2013. Evaluation of the surface urban heat island effect in the city of Madrid by thermal remote sensing. International Journal of Remote Sensing 34, 3177-3192. doi.org/10.1080/01431161.2012.716548

Thenkabail, Ph.D. (Ed.), 2015. Remotely Sensed Data Characterization, Classification, and Accuracies. CRC Press. https://doi.org/10.1201/b19294

Wang, W., Liu, K., Tang, R., Wang, S., 2019. Remote sensing image-based analysis of the urban heat island effect in Shenzhen, China. Physics and Chemistry of the Earth, Parts A/B/C 110, 168-175. doi.org/10.1016/j.pce.2019.01.002
Weng, Q., 2009. Thermal infrared remote sensing for urban climate and environmental studies: Methods, applications, and trends. ISPRS Journal of Photogrammetry and Remote Sensing 64, 335-344. doi.org/10.1016/j.isprsjprs.2009.03.007 